\title{
Computing Blocker Sets for the Regular Post Embedding Problem ${ }^{\star}$
}

\author{
P. Chambart and Ph. Schnoebelen \\ LSV, ENS Cachan, CNRS \\ 61, av. Pdt. Wilson, F-94230 Cachan, France \\ email: \{chambart|phs\}@1sv.ens-cachan.fr
}

\begin{abstract}
Blocker and coblocker sets are regular languages involved in the algorithmic solution of the Regular Post Embedding Problem. We investigate the computability of these languages and related decision problems.
\end{abstract}

\section{Introduction}

Post's Embedding Problem (shortly PEP, named by analogy with Post's Correspondence Problem) is the question whether two morphisms on words $u, v: \Sigma^{*} \rightarrow \Gamma^{*}$ agree non-trivially on some input, i.e., whether $u(x)$ is a (scattered) subword of $v(x)$ for some $x \in \Sigma^{+}$. The subword ordering, also called embedding, is denoted " $\sqsubseteq$ ": $x \sqsubseteq y \stackrel{\text { def }}{\Leftrightarrow} x$ can be obtained from $y$ by erasing some letters, possibly all of them, possibly none.

PEP is trivial if there are no restrictions on the form of solutions. But when one looks for solutions $x$ as above belonging to a regular language $R \subseteq \Sigma^{*}$, the problem (hereafter called the Regular Post Embedding Problem, or PEP ${ }^{\text {reg }}$ ) becomes very interesting: decidable but surprisingly hard [1].

The Regular Post Embedding Problem was introduced in [1,2] where it is shown that PEPreg is expressive enough to encode problems on lossy channel systems. In fact, encodings in both directions exist, hence PEPreg is exactly at level $\mathcal{F}_{\omega^{\omega}}$ in the Fast Growing Hierarchy. Thus, although it is decidable, PEPreg is not primitive-recursive, and not even multiply-recursive (see [3] and the references therein). Finally, PEPreg is an abstract problem that is inter-reducible with a growing list of decidable problems having the same $\mathcal{F}_{\omega^{\omega}}$ complexity: metric temporal logic [4], alternating one-clock timed automata [5,6], leftist grammars [7, 8], products of modal logics [9], etc.

Blockers and coblockers. The original decision algorithm for PEPreg relies on so-called "blocker" and "coblocker" sets [1]. Write $\operatorname{Sol}_{L}$ for the set $\{x \in L \mid u(x) \sqsubseteq v(x)\}$ of solutions in some constraint language $L \subseteq \Sigma^{*}$ and define:

$$
\begin{aligned}
& X_{L} \stackrel{\text { def }}{=}\left\{\alpha \in \Gamma^{*} \mid \forall x \in L, \alpha \cdot u(x) \nsubseteq v(x)\right\}, \\
& X_{L}^{\prime} \stackrel{\text { def }}{=}\left\{\alpha \in \Gamma^{*} \mid \forall x \in L, u(x) . \alpha \nsubseteq v(x)\right\}, \\
& Y_{L} \stackrel{\text { def }}{=}\left\{\alpha \in \Gamma^{*} \mid \forall x \in L, u(x) \nsubseteq \alpha \cdot v(x)\right\}, \\
& Y_{L}^{\prime} \stackrel{\text { def }}{=}\left\{\alpha \in \Gamma^{*} \mid \forall x \in L, u(x) \nsubseteq v(x) . \alpha\right\} .
\end{aligned}
$$

(left $L$-blockers) (right $L$-blockers) (left $L$-coblockers)

(right $L$-coblockers)

\footnotetext{
^ Work supported by the Agence Nationale de la Recherche, grant ANR-06-SETIN-001.
} 
A key observation is that, in order to decide whether $\mathrm{Sol}_{L}$ is empty or not, it is simpler to reason about blocker and coblocker sets (see App. B for more details on the decision algorithm). Rather than considering what are the solutions, the blocker and coblocker sets provide information on what latitude is allowed/required by the solutions, in particular by the most permissive ones. As a special case, they can tell us whether a given PEPreg instance is solvable since

$$
\operatorname{Sol}_{L}=\varnothing \text { iff } \varepsilon \in X_{L} \text { iff } \varepsilon \in X_{L}^{\prime} \text { iff } \varepsilon \in Y_{L} \text { iff } \varepsilon \in Y_{L}^{\prime} .
$$

Working with blocker sets rather than solutions sets has two main advantages:

- First, blocker and coblocker sets behave smoothly as a function of the constraint set $L$. This allows compositional reasoning w.r.t. $L$. The "Stability Inequations" (see App. B) is the main example, but there are more. For instance, assume $L$ is the product (concatenation) of two languages: $L=L_{1} \cdot L_{2}$. Clearly $S_{L} l_{L}$ contains $S l_{L_{1}} . S_{L_{2}}$. However the containment is strict in general, and it is not possible to express $\mathrm{Sol}_{L}$ as a function of $\mathrm{Sol}_{L_{1}}$ and $\mathrm{Sol}_{L_{2}}$. By contrast, the following holds (see App. B):

$$
X_{L_{1} . L_{2}}=\Gamma^{*} \text { iff }\left(X_{L_{1}}^{\prime} \cup Y_{L_{2}}\right) \cap\left(Y_{L_{1}}^{\prime} \cup X_{L_{2}}\right)=\Gamma^{*} .
$$

- Second, blocker and coblocker sets are always regular languages, unlike the $\operatorname{Sol}_{L}$ sets [10]. This makes them easier to handle algorithmically, representing them via FSA's or regular expressions. In particular, compositional reasoning as exemplified in Equation (2) can easily be turned into simple and effective algorithms.

Our contribution. In this paper we consider the computability of the blocker and coblocker sets $X_{R}$ and $Y_{R}$ for $R$ a regular constraint language. This is a natural question in view of the decision algorithm for PEPreg, where lower approximations of these sets are enumerated. More importantly, and as we explain in Section 7, it is another step in our attempts at enlarging the class of known decidable problems that combine Post-embedding and regular constraints.

We prove that blocker sets are not computable ${ }^{1}$ while, quite unexpectedly, coblocker sets are computable. Concerning blocker sets, and since they cannot be computed, we consider decision problems that are weaker than computability, e.g., whether a blocker set is empty, infinite, whether is it contained in ("safety"), or contains ("cosafety"), a given set. A summary of our results will be found in Fig. 3 (section 3). In addition, we answer a question raised by [10] and prove that the regularity of Post-embedding languages is undecidable.

Comparison with existing work. This work continues our exploration of the Regular Post Embedding Problem. The problem was introduced and proved decidable in [1]. The links between lossy channels and PEPreg are clarified in [2] where it is also shown that looking for infinite solutions within an $\omega$-regular constraint set can be reduced to

\footnotetext{
${ }^{1}$ Here, and in the rest of the paper, we say informally that regular sets like $X_{L}$ are "computable" when we really mean that an index for them can be computed uniformly from an index for $L$.
} 
looking for finite solutions. In [10] it is shown how to count solutions, and how to check whether a regular property entails Post embedding. That blocker sets are not computable was claimed in [1, Remark 3.8] without any details or proofs (nor comments on the difference between blocker and coblocker sets).

Outline of the paper. Section 2 recalls the necessary definitions and notations, and proves a few useful lemmas on subwords. Section 3 formally introduces the problems we address. Then Section 4 shows how to compute coblocker sets, while Section 5 considers what can be computed on blocker sets. The undecidability results in that section are proved by a reduction from lossy counter machines described in Section 6. Finally, a technical appendix contains all proofs omitted in the main text.

\section{Notations and definitions}

Words and their morphisms. We write $x, y, w, t, \sigma, \rho, \alpha, \beta, \ldots$ for words, i.e., finite sequences of letters such as $a, b, i, j, \ldots$ from alphabets $\Sigma, \Gamma, \ldots$ With $x . y$, or $x y$, we denote the concatenation of $x$ and $y$. With $\varepsilon$ we denote the empty word. The length of $x$ is written $|x|$.

A morphism from $\Sigma^{*}$ to $\Gamma^{*}$ is a map $u: \Sigma^{*} \rightarrow \Gamma^{*}$ that respects the monoidal structure, i.e., with $u(\varepsilon)=\varepsilon$ and $u(x . y)=u(x) . u(y)$. A morphism $u$ is completely defined by its image $u(a), u(b), \ldots$, on $\Sigma=\{a, b, \ldots\}$. Most of the time, we shall write $u_{a}, u_{b}, \ldots$, and $u_{x}$, instead of $u(a), u(b), \ldots$, and $u(x)$.

The mirror image of a word $x$ is denoted $\widetilde{x}$, e.g., $\widetilde{a b c}=b c a$. The mirror image of a language $L$ is $\widetilde{L} \stackrel{\text { def }}{=}\{\widetilde{x} \mid x \in L\}$. The mirror image of a morphism $u$, denoted $\widetilde{u}$, is defined by $\widetilde{u}(a) \stackrel{\text { def }}{=} \widetilde{u(a)}$, so that $\widetilde{u}(x)=\widetilde{u(\widetilde{x})}$.

Subword ordering. Given two words $x$ and $y$, we write $x \sqsubseteq y$ when $x$ is a (scattered) subword of $y$, i.e., when $x$ can be obtained by erasing some letters (possibly none) from $y$. For example, abba $\underline{a b r a c a d a \underline{b} r a}$. The subword relation is a partial ordering, compatible with the monoidal structure: $\varepsilon \sqsubseteq x$, and $x y \sqsubseteq x^{\prime} y^{\prime}$ when $x \sqsubseteq x^{\prime}$ and $y \sqsubseteq y^{\prime}$. Higman's Lemma further states that, over a finite alphabet, the subword relation is a well-quasi-ordering, i.e., it is well-founded and all antichains (sets of incomparable words) are finite.

Upward-closed and downward-closed languages. A language $L \subseteq \Gamma^{*}$ is upward-closed if $x \in L$ and $x \sqsubseteq y$ imply $y \in L$. It is downward-closed if $x \in L$ and $y \sqsubseteq x$ imply $y \in L$ (equivalently, if its complement is upward-closed). Higman's Lemma entails that upward-closed languages and downward-closed languages are regular [11]. In fact, upward-closed languages can be denoted by very simple regular expressions since they obviously reside at level 1/2 of the Straubing-Thérien Hierarchy [12]. Downwardclosed languages too can be denoted by simple regular expressions [13, 14]. In Section 4 we use "*-products", defined as concatenations of atoms that are either of the form $a+\varepsilon$ for some $a \in \Gamma$, or of the form $A^{*}$ for some sub-alphabet $A \subseteq \Gamma$. For example, with $\Gamma=\{a, b, c\}$, the set of subwords of abac is $(a+\varepsilon) \cdot(b+\varepsilon) \cdot(a+\varepsilon) \cdot(c+\varepsilon)$ and the set of words that do not have $a b$ as a subword is $\{b, c\}^{*} \cdot\{a, c\}^{*}$. Any downward-closed language is, in a unique way, a finite union of maximal $*$-products. 
Combining subwords. We gather a few key properties of the subword ordering in connection with concatenation. Lemma 2.4 is a converse to the Elimination Lemmas.

\section{Fact 2.1}

1. If $x y \sqsubseteq z$, then there exists a factorization $z=z_{1} z_{2}$ of $z$ such that $x \sqsubseteq z_{1}$ and $y \sqsubseteq z_{2}$.

2. If $x \sqsubseteq y z$, then there exists a factorization $x=x_{1} x_{2}$ of $x$ such that $x_{1} \sqsubseteq y$ and $x_{2} \sqsubseteq z$.

Lemma 2.2 (Elimination Lemma). If $x w \sqsubseteq y$ and $x^{\prime} \sqsubseteq w y^{\prime}$ then $x x^{\prime} \sqsubseteq y y^{\prime}$.

Proof. By Fact 2.1 there exist factorizations $y=y_{1} \cdot y_{2}$ and $x^{\prime}=x_{1}^{\prime} \cdot x_{2}^{\prime}$ such that $x \sqsubseteq y_{1}$, $w \sqsubseteq y_{2}, x_{1}^{\prime} \sqsubseteq w$ and $x_{2}^{\prime} \sqsubseteq y^{\prime}$. One concludes with $x x^{\prime}=x x_{1}^{\prime} x_{2}^{\prime} \sqsubseteq y_{1} w y^{\prime} \sqsubseteq y_{1} y_{2} y^{\prime}=y y^{\prime}$.

Lemma 2.3 (Mirror Elimination Lemma). If $x \sqsubseteq y w$ and $w x^{\prime} \sqsubseteq y^{\prime}$ then $x x^{\prime} \sqsubseteq y y^{\prime}$.

Proof. Mirroring the assumptions gives $\widetilde{x} \sqsubseteq \widetilde{w} \widetilde{y}$ and $\widetilde{x^{\prime}} \widetilde{w} \sqsubseteq \widetilde{y^{\prime}}$. Then Lemma 2.2 applies, yielding $\widetilde{x^{\prime}} \widetilde{x} \sqsubseteq \widetilde{y^{\prime}} \widetilde{y}$. Mirroring again gives $x x^{\prime} \sqsubseteq y y^{\prime}$.

Lemma 2.4 (Decomposition Lemma). If $x x^{\prime} \sqsubseteq y y^{\prime}$ then there exists an interpolant $w$ such that

$$
\left(x w \sqsubseteq y \text { and } x^{\prime} \sqsubseteq w y^{\prime}\right) \text { or }\left(x \sqsubseteq y w \text { and } w x^{\prime} \sqsubseteq y^{\prime}\right) .
$$

Proof. Assume $x x^{\prime} \sqsubseteq y y^{\prime}$. Then there exists a factorization $y y^{\prime}=z z^{\prime}$ of $y y^{\prime}$ such that $x \sqsubseteq z$ and $x^{\prime} \sqsubseteq z^{\prime}$ (Fact 2.1). Since $y y^{\prime}=z z^{\prime}$, either $z$ is a prefix of $y$ or $z^{\prime}$ is a suffix of $y^{\prime}$. In the first case, we let the interpolant $w$ be given by writing $y=z w$, so that $z^{\prime}=w y^{\prime}$. Now, from $x \sqsubseteq z$ and $x^{\prime} \sqsubseteq z^{\prime}$, we deduce the required $x w \sqsubseteq y$ and $x^{\prime} \sqsubseteq w y^{\prime}$. In the second case, a mirror reasoning gives $x \sqsubseteq y w$ and $w x^{\prime} \sqsubseteq y^{\prime}$ for $w$ obtained by writing $y^{\prime}=w z^{\prime}$.

\section{Blockers and coblockers}

In the rest of the paper, we consider a generic PEP instance given by some $u, v: \Sigma^{*} \rightarrow$ $\Gamma^{*}$. Recall that, for a regular constraint set $R \subseteq \Sigma^{*}$, the (left) blocker and coblocker sets $X_{R}$ and $Y_{R}$ are defined by:

$$
X_{R} \stackrel{\text { def }}{=}\left\{\alpha \in \Gamma^{*} \mid \forall x \in R, \alpha . u_{x} \nsubseteq v_{x}\right\}, \quad Y_{R} \stackrel{\text { def }}{=}\left\{\alpha \in \Gamma^{*} \mid \forall x \in R, u_{x} \nsubseteq \alpha . v_{x}\right\} .
$$

Observe that $X_{R}$ is upward-closed and $Y_{R}$ is downward-closed. Hence both are regular.

Remark 3.1. In the rest of the paper, starting with Def. 3.2 below, we restrict our attention to the left sets $X_{L}$ and $Y_{L}$. This is no loss of generality in view of the symmetry between the left-handed and the right-handed notions: $\alpha$ is a right $L$-blocker (or coblocker) if, and only if, $\widetilde{\alpha}$ is a left $\widetilde{L}$-blocker (resp., coblocker) in the mirror instance $\widetilde{u}, \widetilde{v}$.

For blocker and coblocker sets, we consider questions that range in generality from just checking one $\alpha$ for membership, to computing the whole set. 
Definition 3.2 (Decision problems for blocker and coblocker sets). We consider questions where one is given two morphisms $u, v: \Sigma^{*} \rightarrow \Gamma^{*}$ and a regular language $R \subseteq \Sigma^{*}$ as inputs, with possibly some additional input in the form of a word $\alpha \in \Gamma^{*}$, or a regular "safe" set $S \subseteq \Gamma^{*}$.

- Blockers_Membership: does $\alpha \in X_{R}$ ?

- Blockers_Emptiness: does $X_{R}=\varnothing$ ?

- Blockers_Universality: does $X_{R}=\Gamma^{*}$ ?

- Blockers_Safety: does $X_{R} \subseteq S$ ?

- Blockers_Cosafety: does $S \subseteq X_{R}$ ?

- Blockers_Finiteness: is $X_{R}$ finite?

- Blockers_Cofiniteness: is $X_{R}$ cofinite?, i.e., is $\Gamma^{*} \backslash X_{R}$ finite?

The same decision problems CoBlockers_Membership, CoBlockers_Safety, ..., are defined for coblocker sets.

Finally, Blockers_Computation and CoBlockers_Computation ask one to compute a representation of $X_{R}$ (resp., $Y_{R}$ ) under the form of a regular expression or a FSA. (These are not decision problems).

Remark 3.3. The restriction to regular safe sets $S$ is a natural assumption that is both expressive and tractable. However, in our setting where blocker and coblocker sets are upward-closed (resp., downward-closed), the expressive power is even larger. Indeed, for any $L, X_{R} \subseteq L$ iff $X_{R} \subseteq S$ where $S$ is the upward-closure of $L$. Thus, and since the upward-closure of $L$ is always regular, our positive results automatically apply to any class of safe sets for which the upward and downward closures can be effectively computed (e.g., context-free languages [15]).

Remark 3.4 (Relations among problems). Safety is a general problem that subsumes Emptiness and Membership. Cosafety subsumes Universality and (non-)Membership. Blockers_Universality reduces to Blockers_Membership since $X_{R}=\Gamma^{*}$ iff $\varepsilon \in X_{R}$. CoBlockers_Universality is trivial since $Y_{R}=\Gamma^{*}$ iff $R=\varnothing$. Finiteness and Cofiniteness are natural counting questions. Finiteness coincides with Emptiness for blocker sets (assuming $\Gamma$ is not empty) and more generally for all upward-closed sets (Cofiniteness and Universality coincide for downward-closed sets in general, and coblocker sets in particular).

There are no other obvious reductions between the above decision problems (e.g., Finiteness and Cofiniteness are in general unrelated).

Regarding computability of the blocker and coblocker sets, observe that since these sets are regular, the decidability of Safety and Cosafety would entail their computability (see also Section 4). Conversely, all the decision problems listed above can easily be answered from an FSA description of the sets. Hence our decision problems can be seen as different special cases of the general Blockers_Computation and CoBlockers_Computation problems.

Remark 3.5 (On the complexity of blocker and coblocker problems). All the non-trivial problems listed in Def. 3.2 are more general than PEPreg. This was made precise in Remark 3.4 except for CoBlockers_Finiteness, but it is easy to provide a reduction from CoBlockers_Emptiness to CoBlockers_Finiteness: add one extra symbol to $\Gamma$, ensuring 


\begin{tabular}{l|c|c|} 
& Blockers & Coblockers \\
\hline \hline Membership & decidable (Coro. 4.2) & decidable (Coro. 4.2) \\
\hline Safety & undecidable (Theo. 5.3) & decidable (Theo. 4.3) \\
\hline Cosafety & decidable (Coro. 4.2) & decidable (Coro. 4.2) \\
\hline Emptiness & undecidable (Theo. 5.3) & decidable (Theo. 4.3) \\
\hline Universality & decidable (Coro. 4.2) & trivial \\
\hline Finiteness & undecidable (Theo. 5.3) & decidable (Theo. 4.3) \\
\hline Cofiniteness & undecidable (Theo. 5.2) & trivial \\
\hline \hline Computation & no & yes (Theo. 4.3) \\
\hline
\end{tabular}

Fig. 1. Computability for blocker and coblocker sets. See Remark 3.5 about complexity.

that $Y_{R}$ is finite iff it is empty. Hence all the above problems are at least as hard as PEP ${ }^{\text {reg }}$ and none of them is multiply-recursive.

\section{Computing coblocker sets}

We start with the computability results. They can be obtained via reductions to PEPreg:

Lemma 4.1. Blockers_Cosafety and CoBlockers_Cosafety many-one reduce to (the complement of) $\mathrm{PEP}{ }^{\mathrm{reg}}$.

Proof. Blockers_Cosafety: with $u, v, R$ and $S$ we associate a PEP ${ }^{\text {reg }}$ instance $u^{\prime}, v^{\prime}$ : $\Sigma^{\prime *} \rightarrow \Gamma^{*}$ and a regular constraint $R^{\prime} \subseteq \Sigma^{*}$. Assume w.l.o.g. that $\Sigma$ and $\Gamma$ are disjoint alphabets and let $\Sigma^{\prime} \stackrel{\text { def }}{=} \Sigma \cup \Gamma$. $u^{\prime}$ and $v^{\prime}$ are extensions of $u$ and $v$ with $u^{\prime}(\gamma)=\gamma$ and $v^{\prime}(\gamma)=\varepsilon$ for all $\gamma \in \Gamma$. Finally let $R^{\prime} \stackrel{\text { def }}{=} S . R$, this is indeed a regular subset of $\Sigma^{\prime *}$.

Now, $u^{\prime}, v^{\prime}, R^{\prime}$ is a positive PEPreg instance iff $u_{x}^{\prime} \sqsubseteq v_{x}^{\prime}$ for some $x \in R^{\prime}$, iff $u_{\alpha y}^{\prime} \sqsubseteq v_{\alpha y}^{\prime}$ for some $\alpha \in S$ and some $y \in R$, iff $u_{\alpha}^{\prime} \cdot u_{y}^{\prime} \sqsubseteq v_{\alpha}^{\prime} \cdot v_{y}^{\prime}$, iff $\alpha . u_{y} \sqsubseteq v_{y}$ for some $\alpha \in S$ and $y$, i.e., iff some $\alpha \in S$ is not in $X_{R}$, i.e., $S \nsubseteq X_{R}$.

CoBlockers_Cosafety: the same idea works provided we let $u^{\prime}(\gamma)=\varepsilon$ and $v^{\prime}(\gamma)=\gamma$.

Note that, since $X_{R}$ is upward-closed, the question whether $S \subseteq X_{R}$ can be reduced to the question whether all the minimal words in $S$ (minimal w.r.t. the subword ordering) belong to $X_{R}$. These minimal words are finitely many (by Higman's Lemma) and it is easy to list them from, e.g., a FSA or a regular expression for $S$. This provides another reduction, albeit not a many-one reduction, from Blockers_Cosafety to Blockers_Membership (or, equivalently, to PEP ${ }^{\text {reg }}$ ).

Since PEPreg is decidable, and thanks to Remark 3.4, Lemma 4.1 entails:

Corollary 4.2. For blocker and coblocker sets, Cosafety, Universality and Membership are decidable.

We are now ready to proceed to the main computability result: 
Theorem 4.3. The coblocker sets $Y_{R}$ and $Y_{R}^{\prime}$ are computable.

Our proof simply leverages the decidability of CoBlockers_Cosafety (Coro. 4.2) with the VJGL Lemma (here specialized to words with embeddings).

Lemma 4.4 (VJGL Lemma, see Theo. 2 of [16]). Let $\left(U_{i}\right)_{i}$ be an enumeration of upward-closed languages on some finite alphabet. One can compute a finite representation for the $U_{i}$ 's if, and only if, one can decide whether $U_{i} \cap P=\varnothing$ for $*$-products $P$ (when $i$ and $P$ are inputs).

Here, computing "a finite representation" means computing the finite basis, i.e, the set of minimal words, but this can easily be transformed into a regular expression or an FSA representation. The VJGL-Lemma is based on a generic algorithm that, in the case of words with embedding, computes such finite bases using an oracle for non-intersection with $*$-products.

Another wording of the VJGL-Lemma is given by the following corollary.

Corollary 4.5. I. If $\left(U_{i}\right)_{i}$ are upward-closed languages with a decidable safety problem, then they are computable.

2. Equivalently, if $\left(V_{i}\right)_{i}$ are downward-closed languages with a decidable cosafety problem, then they are computable.

Proof. $U_{i} \cap P=\varnothing$ is equivalent to $U_{i} \subseteq\left(\Sigma^{*} \backslash P\right)$, a safety question.

We now prove Theorem 4.3: The coblocker sets $Y_{R}$ are downward-closed and have a decidable cosafety problem (Coro. 4.2). Hence they are computable by Coro. 4.5.2. Then Theorem 4.3 accounts for all the positive results on coblocker sets in Fig. 3.

\section{Blocker sets are not computable}

It is not possible to effectively compute the blocker sets $X_{R}$ from given $u, v, R$, even though $X_{R}$ is known to be regular. This is shown with Lemma 5.1, our main negative result (proved in Section 6):

Lemma 5.1. Blockers_Cofiniteness is $\Sigma_{1}^{0}$-hard and Blockers_Emptiness is $\Pi_{1}^{0}$-hard. With Lemma 5.1, we are in a position to prove all the undecidability results in Fig. 3:

Theorem 5.2. Blockers_Cofiniteness is $\Sigma_{1}^{0}$-complete.

Proof (Sketch). Membership in $\Sigma_{1}^{0}$ can be seen by writing the cofiniteness of $X_{R}$ under the form $\exists n \in \mathbb{N}, \Gamma^{\geq n} \subseteq X_{R}$ and relying on the decidability of Blockers_Cosafety (Coro. 4.2).

Theorem 5.3. Blockers_Safety, Blockers_Emptiness and Blockers_Finiteness are $\Pi_{1}^{0}$ complete.

Proof. The $\Pi_{1}^{0}$-hardness of Blockers_Emptiness (Lemma 5.1) also applies to Blockers_Finiteness (since the two problems coincide) and Blockers_Safety (a more general problem), see Remark 3.4.

For upper bounds, we observe that Blockers_Safety (hence also Blockers_Emptiness) is in $\Pi_{1}^{0}$ since it can be written under the form $\forall \alpha \in \Gamma^{*},\left(\alpha \in S \vee \alpha \notin X_{R}\right)$ (recall that $\alpha \notin X_{R}$ is decidable). 


\section{Lossy counter machines}

Lossy counter machines or, for short, LCM's, were introduced by R. Mayr [17]. They are a variant of Minsky counter machines (with zero-test, increments and decrements) where counters are lossy, i.e., they may decrease non-deterministically. We only give a streamlined presentation of LCM's here and refer to $[17,18]$ for more details.

Let $M=\left(Q, C, \Delta, q_{\text {init }}\right)$ be a Minsky counter machine with finite set of control states $Q \ni q_{\text {init }}$, finite set of counters $C$, and finite set of transitions rules $\Delta$. Four counters are sufficient for our purposes so we fix $C=\left\{c_{1}, c_{2}, c_{3}, c_{4}\right\}$. A configuration of $M$ is some $\tau=\left(q, n_{1}, n_{2}, n_{3}, n_{4}\right) \in \operatorname{Conf}(M) \stackrel{\text { def }}{=} Q \times \mathbb{N}^{4}$, with size, denoted $|\tau|$, being $n_{1}+n_{2}+n_{3}+$ $n_{4}$. We (partially) order $\operatorname{Conf}(M)$ with

$$
\left(q, n_{1}, n_{2}, n_{3}, n_{4}\right) \leq\left(q^{\prime}, n_{1}^{\prime}, n_{2}^{\prime}, n_{3}^{\prime}, n_{4}^{\prime}\right) \stackrel{\text { def }}{\Leftrightarrow} q=q^{\prime} \wedge n_{1} \leq n_{1}^{\prime} \wedge \cdots \wedge n_{4} \leq n_{4}^{\prime} .
$$

An initial state $q_{\text {init }} \in Q$ is fixed, and the initial configuration is $\tau_{\text {init }} \stackrel{\text { def }}{=}\left(q_{\text {init }}, 0,0,0,0\right)$. Observe that the only way to have $\tau \leq \tau_{\text {init }}$ is with $\tau=\tau_{\text {init }}$.

A transition rule $\delta$ is a directed edge between states of $M$, labeled by an operation $o p \in O P \stackrel{\text { def }}{=} C \times\{++,--,=0$ ? $\}$, and denoted $\left(q, o p, q^{\prime}\right)$. The rules in $\Delta$ give rise to two different transition relations between configurations. First, steps $\tau \stackrel{\delta}{\rightarrow} \tau^{\prime}$ are defined in the expected way. Formally, with $\delta=\left(q_{1}\right.$, op,$\left.q_{2}\right)$, there is a step $\left(q, n_{1}, n_{2}, n_{3}, n_{4}\right) \stackrel{\delta}{\rightarrow}$ $\left(q^{\prime}, n_{1}^{\prime}, n_{2}^{\prime}, n_{3}^{\prime}, n_{4}^{\prime}\right)$ if, and only if, the following three conditions are satisfied:

1. $q_{1}=q$ and $q_{2}=q^{\prime}$;

2. op is some $c_{k}++$ or $c_{k^{--}}$or $c_{k}=0$ ?, and $n_{i}^{\prime}=n_{i}$ for all $i \neq k$;

3. if $o p$ is $c_{k}++$ then $n_{k}^{\prime}=n_{k}+1$; if op is $c_{k^{--}}$then $n_{k}^{\prime}=n_{k}-1$; if op is $c_{k}=0$ ? then $0=n_{k}=n_{k}^{\prime}$.

These so-called perfect steps describe the operational semantics of $M$ when its counters are not assumed to be lossy. Then a second operational semantics, with transitions denoted $\tau \stackrel{\delta}{\rightarrow}$ lossy $\tau^{\prime}$, is derived ${ }^{2}$ in the following way:

$$
\tau \stackrel{\delta}{\rightarrow} \text { lossy } \tau^{\prime} \stackrel{\text { def }}{\Leftrightarrow} \tau \stackrel{\delta}{\rightarrow} \tau^{\prime \prime} \text { for some } \tau^{\prime \prime} \geq \tau^{\prime}
$$

These lossy steps describe the behavior of $M$ when its counters are assumed to be lossy. In the usual way, the $\delta$ superscript on transitions is omitted when irrelevant. Lossy runs, denoted $\tau_{0} \stackrel{*}{\rightarrow}$ lossy $\tau_{n}$, are sequences of chained lossy steps $\tau_{0} \rightarrow$ lossy $\tau_{1} \rightarrow$ lossy $\cdots \tau_{n}$. We write $\operatorname{Reach}_{\text {lossy }}(M)$ for the set of configurations that can be reached via lossy runs of $M$, starting from $\tau_{\text {init }}$. lems:

We rely on known undecidability results on LCM's and use the following two prob-

LCM_Infinite: the question whether $\operatorname{Reach}_{\text {lossy }}(M)$ is infinite, for a given LCM $M$;

\footnotetext{
${ }^{2}$ Lossy steps could also be defined directly without deriving them from perfect steps, but the indirect definition is very convenient as it permits reasoning simultaneously on both kinds of steps for the same counter machine.
} 
LCM_Unbounded_Counter: the question whether $\operatorname{Reach}_{\text {lossy }}(M)$ contains configurations with arbitrarily large values for the first counter $c_{1}$.

These two problems are a variant of one another, and they are easily seen to be interreducible. The following theorem is from $[17,18]$ :

Theorem 6.1. LCM_Infinite and $L C M \_U n b o u n d e d \_C o u n t e r$ are $\Pi_{1}^{0}$-complete.

\subsection{From lossy counters to Post-embedding}

With a LCM $M=\left(Q, C, \Delta, q_{\text {init }}\right)$ we associate a PEP instance $u, v: \Sigma^{*} \rightarrow \Gamma^{*}$ that will be used in three different reductions (with different constraint languages $R_{1}, R_{2}, R_{3} \subseteq$ $\left.\Sigma^{*}\right)$. Here $\Gamma \stackrel{\text { def }}{=} Q \cup C$ is used to encode the configurations of $M$ : a configuration $\tau=$ $\left(q, n_{1}, n_{2}, n_{3}, n_{4}\right)$ is encoded by the word $c_{1}^{n_{1}} c_{2}^{n_{2}} c_{3}^{n_{3}} c_{4}^{n_{4}} q$, denoted $\lceil\tau\rceil$. Observe that $\lceil\tau\rceil \sqsubseteq$ $\left\lceil\tau^{\prime}\right\rceil$ iff $\tau \leq \tau^{\prime}$.

We further let $\Sigma \stackrel{\text { def }}{=} \Gamma \cup \Delta \cup O P \cup \bar{Q} \cup \bar{C}$ where $\bar{Q}=\{\bar{q} \mid q \in Q\}$ and $\bar{C}=\left\{\bar{c}_{1}, \bar{c}_{2}, \bar{c}_{3}, \bar{c}_{4}\right\}$ are copies of $Q$ and $C$, with new symbols obtained by overlining the original symbols from $Q \cup C$. We define two morphisms $u, v: \Sigma^{*} \rightarrow \Gamma^{*}$ with

$$
\begin{array}{rrrr}
u\left(\left(q, o p, q^{\prime}\right)\right) \stackrel{\text { def }}{=} q, & v\left(\left(q, o p, q^{\prime}\right)\right) \stackrel{\text { def }}{=} q^{\prime}, & u\left(\bar{c}_{i}\right) \stackrel{\text { def }}{=} c_{i}, & v\left(\bar{c}_{i}\right) \stackrel{\text { def }}{=} c_{i}, \\
u\left(c_{i}++\right) \stackrel{\text { def }}{=} \varepsilon, & v\left(c_{i}++\right) \stackrel{\text { def }}{=} c_{i}, & u\left(c_{i} i^{--}\right) \stackrel{\text { def }}{=} c_{i}, & v\left(c_{i}{ }^{--}\right) \stackrel{\text { def }}{=} \varepsilon .
\end{array}
$$

How $u$ and $v$ evaluate on the rest of $\Sigma$ will be defined later when it becomes relevant.

With every transition rule $\delta=\left(q, o p, q^{\prime}\right)$ in $\Delta$, we associate a language $R_{\delta} \subseteq \Sigma^{*}$ given via the following regular expressions:

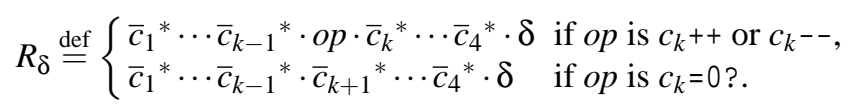

These definitions ensure that, when $x \in R_{\delta}, u_{x}$ and $v_{x}$ are the encodings of related configurations. We let the reader check that the following more precise statement holds:

\section{Lemma 6.2.}

1. If $x \in R_{\delta}$, then $u_{x}=\lceil\tau\rceil$ and $v_{x}=\left\lceil\tau^{\prime}\right\rceil$ for some configurations $\tau, \tau^{\prime}$ such that $\tau \stackrel{\delta}{\rightarrow} \tau^{\prime}$.

2. Reciprocally, if $\tau \stackrel{\delta}{\rightarrow} \tau^{\prime}$, then $\lceil\tau\rceil=u_{x}$ and $\left\lceil\tau^{\prime}\right\rceil=v_{x}$ for some (unique) $x \in R_{\delta}$.

We further define $R_{\Delta} \stackrel{\text { def }}{=} \bigcup_{\delta \in \Delta} R_{\delta}$ and $R_{M} \stackrel{\text { def }}{=}\left(R_{\Delta}\right)^{*}$ : these languages are regular.

Lemma 6.3. Let $\alpha \in \Gamma^{*}$. If $u_{x} . \alpha \sqsubseteq\left\lceil\tau_{\text {init }}\right\rceil . v_{x}$ for some $x \in R_{M}$, then $\alpha \sqsubseteq\lceil\tau\rceil$ for some $\tau \in \operatorname{Reach}_{\text {lossy }}(M)$.

Proof. We assume $\alpha \neq \varepsilon$ and $x \neq \varepsilon$, otherwise $\alpha \sqsubseteq\left\lceil\tau_{\text {init }}\right\rceil$ trivially. Thus $x \in R_{M}$ must be of the form $x=x_{1} \ldots x_{n}$ with $n>0$ and $x_{i} \in R_{\Delta}$ for all $i=1, \ldots, n$. By Lemma 6.2, $u_{x}$ is some $\left\lceil\tau_{0}\right\rceil .\left\lceil\tau_{1}\right\rceil \ldots\left\lceil\tau_{n-1}\right\rceil$ and $v_{x}$ is some $\left\lceil\tau_{1}^{\prime}\right\rceil .\left\lceil\tau_{2}^{\prime}\right\rceil \ldots\left\lceil\tau_{n}^{\prime}\right\rceil$ such that, for all $i=1, \ldots, n$, $\tau_{i-1} \rightarrow \tau_{i}^{\prime}$ is a perfect step of $M$.

We now use the assumption that $u_{x} . \alpha \sqsubseteq\left\lceil\tau_{\text {init }}\right\rceil \cdot v_{x}$. Since $\alpha \neq \varepsilon, u_{x}$ embeds into a strict prefix, denoted $w$, of $\left\lceil\tau_{\text {init }}\right\rceil \cdot v_{x}$. Note that $u_{x}$ contains $n>0$ symbols from $Q$ 
and ends with one of them, while $w$ has at most $n$ (it is shorter than $\left\lceil\tau_{\text {init }}\right\rceil \cdot v_{x}$ that has $n+1$ symbols from $Q$ and ends with one of them). Hence $w$ necessarily has $n$ symbols from $Q$ and $u_{x} . \alpha \sqsubseteq\left\lceil\tau_{\text {init }}\right\rceil . v_{x}$ can be decomposed as $\left\lceil\tau_{i}\right\rceil \sqsubseteq\left\lceil\tau_{i}^{\prime}\right\rceil$ (i.e., $\tau_{i} \leq \tau_{i}^{\prime}$ ) for all $i=1, \ldots, n-1$, with also $\left\lceil\tau_{0}\right\rceil \sqsubseteq\left\lceil\tau_{\text {init }}\right\rceil$ (hence $\tau_{0}=\tau_{\text {init }}$ ) and $\alpha \sqsubseteq\left\lceil\tau_{n}^{\prime}\right\rceil$. Combining with $\tau_{i-1} \rightarrow \tau_{i}^{\prime}$ we deduce $\tau_{i-1} \rightarrow$ lossy $\tau_{i}$ for $i=1, \ldots, n-1$. Finally $\tau_{\text {init }}=\tau_{0} \rightarrow$ lossy $\tau_{1} \cdots \rightarrow \rightarrow_{\text {lossy }} \tau_{n-1} \rightarrow \tau_{n}^{\prime}$ is a lossy run of $M$, so that $\tau_{n}^{\prime} \in \operatorname{Reach}_{\text {lossy }}(M)$.

There is a converse to Lemma 6.3:

Lemma 6.4. If $\tau \in \operatorname{Reach}_{\text {lossy }}(M)$, there exists some $x \in R_{M}$ such that $u_{x} .\lceil\tau\rceil \sqsubseteq\left\lceil\tau_{\text {init }}\right\rceil . v_{x}$.

Proof. Since $\tau \in \operatorname{Reach}_{\text {lossy }}(M)$ there exists a lossy run $\tau_{\text {init }}=\tau_{0} \rightarrow$ lossy $\tau_{1} \rightarrow$ lossy $\cdots \tau_{n}=$ $\tau$. We show, by induction on $i=0,1, \ldots, n$, that $u_{x_{i}} \cdot\left\lceil\tau_{i}\right\rceil \sqsubseteq\left\lceil\tau_{\text {init }}\right\rceil \cdot v_{x_{i}}$ for some $x_{i} \in R_{M}$.

The base case, $i=0$, is dealt with $x_{0}=\varepsilon$ since $\tau_{0}=\tau_{\text {init }}$.

For the case $i>0$, we know by ind. hyp. that there is some $x_{i-1} \in R_{M}$ with

$$
u_{x_{i-1}} \cdot\left\lceil\tau_{i-1}\right\rceil \sqsubseteq\left\lceil\tau_{\text {init }}\right\rceil \cdot v_{x_{i-1}} .
$$

The lossy step $\tau_{i-1} \rightarrow$ lossy $\tau_{i}$ implies the existence of a perfect step $\tau_{i-1} \rightarrow \tau^{\prime}$ with $\tau^{\prime} \geq \tau_{i}$ (Equation (3)). Thus $\left\lceil\tau_{i-1}\right\rceil=u_{y}$ and $\left\lceil\tau^{\prime}\right\rceil=v_{y}$ for some $y \in R_{\Delta}$ (Lemma 6.2).

From $\tau_{i} \leq \tau^{\prime}$, we deduce

$$
u_{y} \cdot\left\lceil\tau_{i}\right\rceil \sqsubseteq\left\lceil\tau_{i-1}\right\rceil \cdot v_{y} .
$$

We now put together Equations (4) and (5). The Elimination Lemma yields

$$
u_{x_{i-1}} \cdot u_{y} \cdot\left\lceil\tau_{i}\right\rceil \sqsubseteq\left\lceil\tau_{\text {init }}\right\rceil \cdot v_{x_{i-1}} \cdot v_{y}
$$

so that setting $x_{i} \stackrel{\text { def }}{=} x_{i-1} . y$ concludes our proof. We observe that $x_{i} \in R_{M}$ since $x_{i-1} \in R_{M}$ and $y \in R_{\Delta}$.

\subsection{Reducing LCM_Infinite and LCM_Unbounded_Counter to blockers problems}

For the next step in the reduction, we extend $u$ and $v$ on $Q \cup C(=\Gamma)$ with

$$
u(\gamma) \stackrel{\text { def }}{=} \pi_{1}(\gamma)=\left\{\begin{array}{ll}
c_{1} & \text { if } \gamma=c_{1}, \\
\varepsilon & \text { if } \gamma \in \Gamma \backslash\left\{c_{1}\right\},
\end{array} \quad v(\gamma) \stackrel{\text { def }}{=} \gamma \text { for all } \gamma \in \Gamma .\right.
$$

When $\alpha \in \Gamma^{*}$, we shall write $\pi_{1}(\alpha)$ rather than $u_{\alpha}$ to emphasize the fact that $u$ only retains the $c_{1}$ symbols of $\alpha$ and erases the rest. Below, we rely on a few obvious properties of this erasing morphism, such as $\pi_{1}(\alpha) \sqsubseteq \alpha$, or $\pi_{1}(\alpha \beta)=\pi_{1}(\beta \alpha)$, and in particular the following:

Fact 6.5 For all $\beta \in \Gamma^{*}$ and $x, y \in \Sigma^{*}, x . c_{1} . \pi_{1}(\beta) \sqsubseteq y . \beta$ implies $x . c_{1} \sqsubseteq y$.

Finally, we let $R_{1} \stackrel{\text { def }}{=} q_{\text {init }} \cdot R_{M}$ and $R_{2} \stackrel{\text { def }}{=} R_{1} \cdot \Gamma^{*}$. This provides two different reductions, with properties captured by Lemmas 6.6 and 6.8 . 
Lemma 6.6. Let $\alpha \in \Gamma^{*}$. The following are equivalent:

(1) $\alpha \notin X_{R_{1}}^{\prime}$,

(2) there exists $x \in R_{1}$ such that $u_{x} . \alpha \sqsubseteq v_{x}$,

(3) there exists $\tau \in$ Reach $_{\text {lossy }}(M)$ such that $\alpha \sqsubseteq\lceil\tau\rceil$.

Proof (Sketch). (1) $\Leftrightarrow$ (2) by definition of $X_{R_{1}}^{\prime}$. Then, given the definitions of $R_{1}, u$ and $v$, Lemma 6.3 shows “(2) $\Rightarrow(3)$ " (note that $u\left(q_{\text {init }}\right)=\varepsilon$ and $v\left(q_{\text {init }}\right)=q_{\text {init }}=\left\lceil\tau_{\text {init }}\right\rceil$ ). Finally, Lemma 6.4 shows “(3) $\Rightarrow(2)$ ”.

In particular, $X_{R_{1}}^{\prime}$ is cofinite iff $M$ does not satisfy LCM_Infinite.

Corollary 6.7. Blockers_Cofiniteness is $\Sigma_{1}^{0}$-hard.

Lemma 6.8. Let $\alpha \in \Gamma^{*}$. The following are equivalent:

(1) $\alpha \notin X_{R_{2}}^{\prime}$,

(2) there exists $y \in R_{2}$ such that $u_{y} . \alpha \sqsubseteq v_{y}$,

(3) there exists $\tau \in$ Reach $_{\text {lossy }}(M)$ such that $\pi_{1}(\alpha) \sqsubseteq \pi_{1}(\lceil\tau\rceil)$.

Proof. (1) $\Leftrightarrow$ (2) by definition of $X_{R_{2}}^{\prime}$.

(3) $\Rightarrow(2)$ : Assume $\pi_{1}(\alpha) \sqsubseteq \pi_{1}(\lceil\tau\rceil)$ for some $\tau \in \operatorname{Reach}_{\text {lossy }}(M)$. Then, $\pi_{1}(\alpha) \sqsubseteq\lceil\tau\rceil$ so that, by Lemma 6.6, there exists some $x \in R_{1}$ with $u_{x} \cdot \pi_{1}(\alpha) \sqsubseteq v_{x}$. Appending $\alpha$ to the right yields $u_{x} . \pi_{1}(\alpha) \cdot \alpha=u_{x} \cdot u_{\alpha} \cdot \alpha \sqsubseteq v_{x} \cdot \alpha=v_{x} \cdot v_{\alpha}$. Letting $y \stackrel{\text { def }}{=} x . \alpha\left(\in R_{2}\right)$ proves (2). (2) $\Rightarrow$ (3): Assume $u_{y} . \alpha \sqsubseteq v_{y}$ for some $y \in R_{2}$ of the form $x . \beta$ with $x \in R_{1}$ and $\beta \in \Gamma^{*}$. We assume $\pi_{1}(\alpha) \neq \varepsilon$ since otherwise $\pi_{1}(\alpha) \sqsubseteq \pi_{1}\left(\left\lceil\tau_{\text {init }}\right\rceil\right)$ holds trivially. From $u_{y} . \alpha \sqsubseteq v_{y}$, we deduce

$$
u_{x} \cdot \pi_{1}(\alpha) \cdot \pi_{1}(\beta)=u_{x} \cdot \pi_{1}(\beta) \cdot \pi_{1}(\alpha)=u_{y} \cdot \pi_{1}(\alpha) \sqsubseteq u_{y} \cdot \alpha \sqsubseteq v_{y}=v_{x} \cdot v_{\beta}=v_{x} \cdot \beta .
$$

From $u_{x} . \pi_{1}(\alpha) \cdot \pi_{1}(\beta) \sqsubseteq v_{x} . \beta$, one deduces $u_{x} . \pi_{1}(\alpha) \sqsubseteq v_{x}$ (using Fact 6.5 and the assumption that $\left.\pi_{1}(\alpha) \neq \varepsilon\right)$. Thus there exists a $\tau \in \operatorname{Reach}_{\text {lossy }}(M)$ with $\pi_{1}(\alpha) \sqsubseteq\lceil\tau\rceil$ (Lemma 6.3), hence $\pi_{1}(\alpha) \sqsubseteq \pi_{1}(\lceil\tau\rceil)$.

In other words, $\alpha \notin X_{R_{2}}^{\prime}$ iff there is a reachable configuration where the $c_{1}$ counter is larger than, or equal to, the number of $c_{1}$ symbols in $\alpha$. Thus $X_{R_{2}}^{\prime}=\varnothing$ iff $M$ satisfies LCM_Unbounded_Counter.

Corollary 6.9. Blockers_Emptiness is $\Pi_{1}^{0}$-hard.

As an aside, the reduction from LCM's can be used to prove Theo. 6.11 below. The regularity problem for Post-embedding languages is a natural question since $\operatorname{Sol}_{R}$ is not always regular, and since comparisons with a regular $S$ are possible:

Theorem 6.10 ([10]). The questions, for $S \subseteq \Sigma^{*}$ a regular language, whether $S \subseteq$ Sol $_{R}$, and whether $\operatorname{Sol}_{R} \subseteq S$, are decidable.

Theorem 6.11 (See App. A). The question whether, for $u, v: \Sigma^{*} \rightarrow \Gamma^{*}$ and a regular $R \subseteq \Sigma^{*}$, Sol $_{R}$ is a regular language, is $\Sigma_{1}^{0}$-complete.

The proof for $\Sigma_{1}^{0}$-hardness simply adapts our previous reduction, providing $u, v$ and $R$ such that $\operatorname{Sol}_{R}$ is regular iff $\operatorname{Reach}_{\text {lossy }}(M)$ is finite, then relying on Theo. 6.1. 


\section{Concluding remarks}

The decidability of PEPreg is the decidability of existential questions of the form

$$
\exists x \in R: u(x) \sqsubseteq v(x)
$$

for regular $R$ 's. This result is fragile and does not extend easily. When one looks for solutions satisfying more expressive constraints, e.g., deterministic context-free, or also Presburger-definable, the problem becomes undecidable [1]. In another direction, combining two embeddings quickly raises undecidable questions, e.g., the following questions are undecidable [10, Theo. 4.1]:

$$
\begin{aligned}
& \exists x \in \Sigma^{+}:\left(u_{1}(x) \sqsubseteq v_{1}(x) \wedge u_{2}(x) \sqsubseteq v_{2}(x)\right), \\
& \exists x \in \Sigma^{+}:\left(u_{1}(x) \sqsubseteq v_{1}(x) \wedge u_{2}(x) \nsubseteq v_{2}(x)\right) .
\end{aligned}
$$

Remark that, by Theorem 6.10, the following universal question is decidable [10]:

$$
\forall x \in R: u(x) \sqsubseteq v(x) .
$$

This suggests considering questions like

$$
\begin{aligned}
& \forall x \in R \exists x^{\prime} \in R^{\prime}: u\left(x x^{\prime}\right) \sqsubseteq v\left(x x^{\prime}\right), \\
& \exists x \in R \forall x^{\prime} \in R^{\prime}: u\left(x x^{\prime}\right) \sqsubseteq v\left(x x^{\prime}\right) .
\end{aligned}
$$

The undecidability of (Q5) is clear since already Blockers_Emptiness is undecidable. The (un?)decidability of (Q6) is still open. We believe blockers and coblockers may play a useful role here. Indeed, by analogy with blockers, we may define

$$
A_{R} \stackrel{\text { def }}{=}\{\alpha \mid \forall x \in R, \alpha \cdot u(x) \sqsubseteq v(x)\}, \quad B_{R} \stackrel{\text { def }}{=}\{\beta \mid \forall x \in R, u(x) \sqsubseteq \beta . v(x)\} .
$$

Note that membership in $A_{R}$ (or in $B_{R}$ ), being an instance of (Q5), is decidable. Furthermore, $B_{R}$ is upward-closed and $A_{R}$ is finite (unless $R$ is empty). Now, the following observation:

$$
\left(\exists x \in R \forall x^{\prime} \in R^{\prime}: u\left(x x^{\prime}\right) \sqsubseteq v\left(x x^{\prime}\right)\right) \text { iff }\left(\left(A_{R^{\prime}} \backslash Y_{R}\right) \cup\left(B_{R^{\prime}} \backslash X_{R}\right) \neq \varnothing\right)
$$

provides a direct link between (Q6) and blocker-like languages. We leave this as a suggestion for future investigations.

\section{Acknowledgements}

We thank Sylvain Schmitz for his numerous remarks and suggestions about this work.

\section{References}

1. P. Chambart and $\mathrm{Ph}$. Schnoebelen. Post embedding problem is not primitive recursive, with applications to channel systems. In Proc. FST\&TCS 2007, volume 4855 of Lecture Notes in Computer Science, pages 265-276. Springer, 2007. 
2. P. Chambart and Ph. Schnoebelen. The $\omega$-Regular Post Embedding Problem. In Proc. FOSSACS 2008, volume 4962 of Lecture Notes in Computer Science, pages 97-111. Springer, 2008.

3. P. Chambart and Ph. Schnoebelen. The ordinal recursive complexity of lossy channel systems. In Proc. LICS 2008, pages 205-216. IEEE Comp. Soc. Press, 2008.

4. J. Ouaknine and J. Worrell. On the decidability and complexity of Metric Temporal Logic over finite words. Logical Methods in Comp. Science, 3(1):1-27, 2007.

5. P. A. Abdulla, J. Deneux, J. Ouaknine, K. Quaas, and J. Worrell. Universality analysis for one-clock timed automata. Fundamenta Informaticae, 89(4):419-450, 2008.

6. S. Lasota and I. Walukiewicz. Alternating timed automata. ACM Trans. Computational Logic, 9(2), 2008.

7. T. Jurdziński. Leftist grammars are nonprimitive recursive. In Proc. ICALP 2008, volume 5126 of Lecture Notes in Computer Science, pages 51-62. Springer, 2008.

8. P. Chambart and $\mathrm{Ph}$. Schnoebelen. Toward a compositional theory of leftist grammars and transformations. In Proc. FOSSACS 2010, volume 6014 of Lecture Notes in Computer Science, pages 237-251. Springer, 2010.

9. D. Gabelaia et al. Non-primitive recursive decidability of products of modal logics with expanding domains. Annals of Pure and Applied Logic, 142(1-3):245-268, 2006.

10. P. Chambart and Ph. Schnoebelen. Pumping and counting on the Regular Post Embedding Problem. In Proc. ICALP 2010, Lecture Notes in Computer Science. Springer, 2010.

11. L. H. Haines. On free monoids partially ordered by embedding. J. Combinatorial Theory, 76:94-98, 1969.

12. J.-E. Pin and P. Weil. Polynomial closure and unambiguous product. Theory of Computing Systems, 30(4):383-422, 1997.

13. A. Finkel and J. Goubault-Larrecq. Forward analysis for WSTS, part I: Completions. In Proc. STACS 2009, Leibniz International Proceedings in Informatics, pages 433-444, 2009.

14. P. A. Abdulla, A. Collomb-Annichini, A. Bouajjani, and B. Jonsson. Using forward reachability analysis for verification of lossy channel systems. Formal Methods in System Design, 25(1):39-65, 2004.

15. J. van Leeuwen. Effective constructions in well-partially-ordered free monoids. Discrete Mathematics, 21(3):237-252, 1978.

16. J. Goubault-Larrecq. On a generalization of a result by Valk and Jantzen. Research Report LSV-09-09, Laboratoire Spécification et Vérification, ENS Cachan, France, May 2009.

17. R. Mayr. Undecidable problems in unreliable computations. Theoretical Computer Science, 297(1-3):337-354, 2003.

18. Ph. Schnoebelen. Lossy counter machines: A survey. In Proc. RP 2010, Lecture Notes in Computer Science. Springer, 2010. 


\section{A Regularity of Post-embedding languages is undecidable}

In this section we prove one half of Theorem 6.11, i.e., that the regularity of $\mathrm{Sol}_{R}$ is $\Sigma_{1}^{0}$-hard. The other half, membership in $\Sigma_{1}^{0}$, is a consequence of Theorem 6.10.

We consider the reduction from LCM_Infinite to PEP built in Section 6.1 and further extend $u$ and $v$ on $\bar{Q}$ with $u(\bar{q})=q$ and $v(\bar{q})=\varepsilon$ for each $\bar{q} \in \bar{Q}$. We further define $R_{3}=q_{\text {init }} \cdot R_{M} \cdot \bar{Q}$. In this framework, the following holds:

Lemma A.1. If Reach lossy $(M)$ is finite, then Sol $_{R_{3}}$ is regular.

Proof (Sketch). Any $x \in R_{3}$ has the form $q_{\text {init }} \cdot x_{1} \cdot x_{2} \ldots x_{n} \cdot \bar{q}$ for some $q \in Q$ and some $x_{1}, x_{2}, \ldots, x_{n} \in R_{\Delta}$. As seen in the proof of Lemma 6.3, such an $x$ belongs to $\operatorname{Sol}_{R_{3}}$ if, and only if, there exists a lossy run

$$
\left(\tau_{\text {init }}=\right) \tau_{0} \stackrel{\delta_{1}}{\rightarrow} \text { lossy } \tau_{1} \stackrel{\delta_{2}}{\rightarrow} \text { lossy } \cdots \stackrel{\delta_{n}}{\rightarrow} \text { lossy } \tau_{n}
$$

with $x_{i} \in R_{\delta_{i}}, u_{x_{i}}=\left\lceil\tau_{i-1}\right\rceil$ (and where the control state of $\tau_{n}$ is $q$ ). The assumption that $\operatorname{Reach}_{\text {lossy }}(M)$ is finite implies that the set of lossy runs in $(*)$, when viewed as sequences $\sigma$ of the form $\left(\tau_{0}, \delta_{1}\right) \ldots\left(\tau_{n-1}, \delta_{n}\right)$ over the (finite!) alphabet $\operatorname{Reach}_{\text {lossy }}(M) \times$ $\Delta$, is a regular language, as is the set of paths of any finite graph. Since there is a bijective correspondence between the $x_{i}$ 's and the pairs $\left(\tau_{i-1}, \delta_{i}\right)$ (see Lemma 6.2), the set of all $x_{1} \ldots x_{n}$ that correspond to lossy runs is regular too, hence also $\mathrm{Sol}_{R_{3}}$.

We can prove a reciprocal of Lemma A.1 if we restrict ourselves to deflatable counter machines. Formally, a counter machine $M$ is deflatable if it contains among its transition rules, the so-called "deflating" rules $q \stackrel{c^{i^{--}}}{\longrightarrow} q$ for all states $q \in Q$ and counters $c_{i} \in C$.

Lemma A.2. If Reach lossy $_{(M)}$ is infinite and $M$ is deflatable, then Sol $_{R_{3}}$ is not regular.

Proof (Sketch). For the proof, we use the projection morphism $\pi_{\Delta}: \Sigma^{*} \rightarrow \Delta^{*}$ that erases all symbols not in $\Delta$ (recall that, in our reduction from LCM's to PEP, the set of rules $\Delta$ is a sub-alphabet of $\Sigma)$ and we show that $\pi_{\Delta}\left(\operatorname{Sol}_{R_{3}}\right)$ is not regular, which is sufficient since morphisms preserve regularity.

Now, since $\operatorname{Reach}_{\text {lossy }}(M)$ is infinite, for every $N \in \mathbb{N}$ there exists a reachable configuration $\tau_{N}$ having size $N$. From $\tau_{N}, N$ deflating steps are possible and not more. Thus, for each $N \in \mathbb{N}$, there is a lossy run of the form

$$
\tau_{0} \stackrel{\delta_{N, 1}}{\longrightarrow} \text { lossy } \cdots \stackrel{\delta_{N, k_{N}}}{\longrightarrow} \text { lossy } \tau_{N} \underbrace{\stackrel{\text { defl }}{\longrightarrow} \text { lossy } \cdots \stackrel{\text { defl }}{\longrightarrow} \text { lossy }}_{N \text { deflating steps }} \tau_{N}^{\prime} .
$$

With this lossy run one associates a word $y_{N} \in R_{3}$, exactly as in the proof of Lemma A.1. Now $\pi_{\Delta}\left(y_{N}\right)$ is $\delta_{N, 1} \ldots \delta_{N, k_{N}}(\text { defl })^{N}$, i.e., some $Y_{N}(\text { defl })^{N}$ (for simplifying purposes, we assume $\pi_{\Delta}$ further projects all different deflating rules to a single one called just "defl").

If $\pi_{\Delta}\left(\mathrm{Sol}_{R_{3}}\right)$ is regular, the pumping lemma for regular language implies that, for $N$ large enough, if $\pi_{\Delta}\left(\operatorname{Sol}_{R_{3}}\right)$ contains $Y_{N}(\operatorname{defl})^{N}$, it also contains $Y_{N}(\operatorname{defl})^{N}\left(\operatorname{defl}^{m}\right)^{*}$ for some $m>0$. But this is clearly impossible since it would imply the existence of lossy runs starting with the same $k_{N}$ steps and ending with arbitrarily many deflating steps.

Hence neither $\pi_{\Delta}\left(\operatorname{Sol}_{R_{3}}\right)$, nor $\operatorname{Sol}_{R_{3}}$, are regular. 
We conclude by observing that the restriction to deflatable counter machines is no loss of generality. Deflating rules mimic losses in counters, hence any counter machine can be turned into a deflatable one that has essentially the same behavior as long as one only considers the lossy semantics. In particular, the original machine and its deflatable version have exactly the same reachable configurations (via lossy runs).

Therefore, Lemmas A.1 and A.2 show that LCM_Infinite reduces to the question whether the solutions of a PEPreg instance is a regular language. Hence the regularity of $\operatorname{Sol}_{R}$ is $\Sigma_{1}^{0}$-hard as announced.

\section{B Solving PEPreg with blockers and coblockers}

We start with a warm-up exercise: proving Equation (2) from the introduction. The proof is clearer if the equation is written contrapositionally, under the form:

$$
X_{L_{1} . L_{2}} \neq \Gamma^{*} \text { iff }\left[X_{L_{1}}^{\prime} \cup Y_{L_{2}} \neq \Gamma^{*} \text { or } Y_{L_{1}}^{\prime} \cup X_{L_{2}} \neq \Gamma^{*}\right] \text {. }
$$

Proof. ( $\Leftarrow$ :) assume that there exists some $\alpha \in \Gamma^{*}$ that does not belong to $X_{L_{1}}^{\prime} \cup Y_{L_{2}}$ (the case $\alpha \notin Y_{L_{1}}^{\prime} \cup X_{L_{2}}$ is symmetric). Hence $\alpha \notin X_{L_{1}}^{\prime}$ and $\alpha \notin Y_{L_{2}}$. Therefore $u_{x_{1}} . \alpha \sqsubseteq v_{x_{1}}$ for some $x_{1} \in L_{1}$, and $u_{x_{2}} \sqsubseteq \alpha \cdot v_{x_{2}}$ for some $x_{2} \in L_{2}$. We deduce $u_{x_{1}} u_{x_{2}} \sqsubseteq v_{x_{1}} v_{x_{2}}$ (by the Elimination Lemma). Hence taking $x=x_{1} x_{2}$ shows $\varepsilon \notin X_{L_{1} . L_{2}}$ and then $X_{L_{1} . L_{2}} \neq \Gamma^{*}$.

( $\Rightarrow$ :) If $X_{L_{1} . L_{2}} \neq \Gamma^{*}$ then, in particular, $\varepsilon \notin X_{L_{1} . L_{2}}$ (since blocker sets are upward-closed) and there exists some $x \in L_{1} \cdot L_{2}$ with $u_{x} \sqsubseteq v_{x}$. Writing $x$ under the form $x=x_{1} x_{2}$ with $x_{1} \in L_{1}$ and $x_{2} \in L_{2}$, we deduce $u_{x_{1}} \cdot u_{x_{2}} \sqsubseteq v_{x_{1}} \cdot v_{x_{2}}$. Thus, by Lemma 2.4 (Decomposition Lemma), there exists $w \in \Gamma^{*}$ such that either $u_{x_{1}} \cdot w \sqsubseteq v_{x_{1}}$ and $u_{x_{2}} \sqsubseteq w \cdot v_{x_{2}}$, or $u_{x_{1}} \sqsubseteq v_{x_{1}} \cdot w$ and $w \cdot u_{x_{2}} \sqsubseteq v_{x_{2}}$. In the first case, $w \notin X_{L_{1}}^{\prime}$ and $w \notin Y_{L_{2}}$. In the second case $w \notin Y_{L_{1}}^{\prime}$ and $w \notin X_{L_{2}}$. Thus $X_{L_{1}}^{\prime} \cup Y_{L_{2}} \neq \Gamma^{*}$ or $Y_{L_{1}}^{\prime} \cup X_{L_{2}} \neq \Gamma^{*}$.

We now recall the ideas behind the proof (in [1]) that PEPreg is decidable. With Equation (1) above, PEP reg reduces to whether the blocker/coblocker sets $X_{R}$ and $Y_{R}$ contain the word $\varepsilon$. Hence it certainly suffices to show that one can decide whether a given $\alpha$ belongs to some $X_{R}$ (or to some $Y_{R}$ ) for a regular language $R \subseteq \Sigma^{*}$, two problems called Blockers_Membership and, respectively, CoBlockers_Membership in Def. 3.2.

The blocker sets $X_{L}$ and the coblocker sets $Y_{L}$ are related to blocker and coblocker sets $\left(X_{a^{-1} L}, Y_{a^{-1} L}\right)_{a \in \Sigma}$ where, for $u \in \Sigma^{*}, u^{-1} L$ denotes the (right) quotient of $L$ by $u$ :

$$
u^{-1} L \stackrel{\text { def }}{=}\left\{w \in \Sigma^{*} \mid u . w \in L\right\} .
$$

More precisely, it is shown in [1] that the pairs $\left(X_{L}, Y_{L}\right)_{L \subset \Sigma^{*}}$ are the largest solutions of a family of mutually recursive inequations of the form

$$
\left(A_{L}, B_{L}\right) \subseteq f\left[\left(A_{a^{-1} L}, B_{a^{-1} L}\right)_{a \in \Sigma}\right]
$$

(Stability)

where $f$ is a $k$-ary (for $k=|\Sigma|$ ) monotonic function on pairs of languages. In the case of a regular language $R \subseteq \Sigma^{*}$, the Myhill-Nerode Theorem states that the set of its quotients 
$Q u o(R) \stackrel{\text { def }}{=}\left\{w^{-1} R \mid w \in \Sigma^{*}\right\}$ is a finite set of regular languages. ${ }^{3}$ Thus $X_{R}$ and $Y_{R}$ can be defined as the largest solutions of a finite number of stability inequations that involve finitely many parameters, namely all $\left\{A_{R^{\prime}}, B_{R^{\prime}} \mid R^{\prime} \in Q u o(R)\right\}$.

Additionally, $f$ is regularity-preserving, and its image can be effectively computed from its arguments $\left(A_{a^{-1} R}, B_{a^{-1} R}\right)_{a \in \Sigma}$ when they are regular languages (see [1, Section 3]). This implies the following:

Corollary B.1 ([1]). The questions whether $\alpha \in X_{R}$ or $\alpha \in Y_{R}$ for $R \subseteq \Sigma^{*}$ a regular constraint language are recursively enumerable.

Proof (Idea). One can enumerate all families $\left(A_{R^{\prime}}, B_{R^{\prime}}\right)_{R^{\prime} \in Q u o(R)}$ of pairs of regular languages indexed by quotients of $R$ and effectively check for stability. When a stable family is encountered, any $\alpha$ in $A_{R}$ (or in $B_{R}$ ) is a blocker (resp., a coblocker) since $X_{R} \supseteq A_{R}$ and $Y_{R} \supseteq B_{R}$ by virtue of being the largest solution. Furthermore, any blocker or coblocker $\alpha$ will eventually be found by this method since the largest stable family, consisting of the actual $\left(X_{R^{\prime}}, Y_{R^{\prime}}\right)_{R^{\prime} \in Q u o(R)}$ will appear in the enumeration.

With Corollary B.1 decidability of Blockers_Membership and CoBlockers_Membership ensues since not being a blocker (or a coblocker) is evidently recursively enumerable: it suffices to guess some $x \in R$ and check that $\alpha . u_{x} \sqsubseteq v_{x}$ (or, respectively, that $\left.u_{x} \sqsubseteq \alpha . v_{x}\right)$.

\footnotetext{
${ }^{3}$ Furthermore, $Q u o(R)$ is easily obtained from a canonical DFSA for $R$ by varying the initial state.
} 